\title{
Endoscopic Ultrasound-Guided Fine Needle Aspiration in the Diagnosis of Mediastinal Masses of Unknown Origin
}

Marc F. Catalano, M.D., Mark L. Rosenblatt, M.D., Amitabh Chak, M.D., Michael V. Sivak, Jr., M.D., James Scheiman, M.D., and Frank Gress, M.D.

St. Luke's Medical Center, Pancreatic Biliary Center, Milwaukee, Wisconsin; University Hospitals of Cleveland, Cleveland, Ohio; University of Michigan, Ann Arbor, Michigan; Winthrop Hospital, Long Island,

New York

OBJECTIVES: The ability of endosonography to diagnose a variety of gastrointestinal pathology has been significantly advanced with the introduction of endoscopic ultrasoundguided fine needle aspiration (EUS-FNA) biopsy. EUSFNA technology can also be applied to the evaluation of non-GI disorders. The role of EUS-FNA to establish the diagnosis of unexplained mediastinal masses has not been previously described. The aim of this study was to determine the diagnostic accuracy, impact on subsequent workup, and role of EUS-FNA in treating mediastinal masses of unknown cause.

METHODS: A total of 26 patients (15 men and 11 women, mean age $61 \mathrm{yr}$, range 39-77 yr) underwent EUS-FNA in patients presenting with unexplained mediastinal masses at four tertiary referral centers. Presenting symptoms included: chest pain (10 patients), dysphagia (eight), cough (seven), fever (six), night sweats (three), and no symptoms/abnormal x-ray (five patients). Five of 26 patients had prior history of cancer (three lung, one tracheal, and one esophageal).

RESULTS: Final diagnosis using EUS-FNA, surgery, autopsy, other diagnostic study, or long-term follow-up was available in all patients. EUS-FNA results were classified under three disease categories: 1) infectious, 2) benign/ inflammatory, and 3) malignant. Final diagnosis included infectious in five patents, benign/inflammatory in nine, and malignant in 12. EUS-FNA was successful in 21 of 26 patients $(81 \%)$ for all disease categories (infectious $60 \%$, benign/inflammatory $78 \%$, and malignant $92 \%$ ). EUS-FNA was successful in directing subsequent workup in 77\% (20 of 26) and therapy in $73 \%$ (19 of 26). Mean EUS-FNA passes for adequate tissue sampling was lower of nonmalignant disease categories (3.0 and 3.4) versus malignant disease (4.4). No complications were seen during the course of this study.

CONCLUSIONS: EUS-FNA in patients presenting with idiopathic mediastinal masses establishes the diagnosis in the vast majority of cases, particularly for those with malignant disease. The emergence of transesophageal EUS-FNA of the mediastinum provides the ability to alter subsequent workup and therapy, obviating the need for more invasive diagnostic studies such as thoracotomy. (Am J Gastroenterol 2002;97: 2559-2565. (C) 2002 by Am. Coll. of Gastroenterology)

\section{INTRODUCTION}

Thoracic imaging of mediastinal masses is challenging. CT of the chest is the current standard by which the mediastinum is visualized. Problems then arise as to various tissue sampling modalities. In well-selected patients, CT-guided fine needle aspiration (FNA), mediastinoscopy, thoracoscopic mediastinal biopsy, or transbronchial biopsy has been used. Morbidity, time, and costs have limited their applicability.

The advent of endoscopic ultrasonography (EUS) has made it possible to image the GI tract and surrounding extraluminal tissues. EUS-FNA has become the preferred method for staging of upper GI tract cancers. The esophagus affords an excellent acoustic window through which to scan the mediastinum, a fact that extends the usefulness of this examination. Recently, EUS-FNA has been shown to have a valuable role in identifying posterior mediastinal involvement when staging nongastrointestinal malignancies $(1,2)$.

The purpose of this study was to assess the diagnostic accuracy of EUS-FNA on a heterogenous group of patients presenting with idiopathic mediastinal mass lesions, and to assess the impact of EUS-FNA on subsequent workup and therapy.

\section{MATERIALS AND METHODS}

A total of 26 patients underwent EUS-FNA in patients presenting with idiopathic and mediastinal masses at four tertiary referral centers. This group included 15 men and 11 women, with a mean age of $61 \mathrm{yr}$ (range 38-77 yr). Inclusion criteria included age $\geq 18 \mathrm{yr}$ with informed consent, absence of tissue diagnosis at the time of EUS, and final diagnosis established. Exclusion criteria included previ- 
ously established diagnosis, inaccessible masses for EUSFNA, coagulopathy, or final diagnosis not available at follow-up. CT was obtained in all patients before referral for EUS, and was abnormal (demonstrating a mediastinal mass in the absence of lymphadenopathy) in 23 of 26 patients $(89 \%)$. The remaining three patients had incidental mass found within the mediastinum on EUS

Presenting symptoms included chest pain in 10 patients, dysphagia in eight, cough in seven, fever in six, night sweats in three, and no symptoms/abnormal x-ray in five. Five of 26 patients had a prior history of cancer, including three patients with lung cancer, one with laryngeal cancer, and one with esophageal cancer, but were believed to be free of disease after surgical therapy (16 months to $3.5 \mathrm{yr}$ before EUS). Final diagnosis using EUS-FNA, surgery, autopsy, and other diagnostic study or long-term follow-up was available in all patients. EUS FNA results were classified under three disease categories: 1) infectious, 2) benign/ inflammatory, and 3) malignant mediastinal.

All patients underwent radial endosonography (Olympus EUM-20, Olympus America, Lake Success, NY) followed by linear array endosonography (Pentax FG32-UA, Pentax, Orangeburg, NY, or Olympus GFUC-30P). Fine needle aspiration was accomplished using two different needle types: a 22-gauge, 8 -cm, adjustable length needle (WilsonCook, Winston-Salem, NC) and a 22-gauge, 12-cm, adjustable length needle (GIP, Medi-Globe, Tempe, AZ). A cytopathologist was present in all cases to evaluate adequacy of each EUS-FNA sample.

The impact of EUS-FNA results in terms of changing clinical management (theoretic $v s$ actual plan), including eventual workup and treatment, was determined by each endoscopist for each individual case. The change in clinical management definition was the difference between the theoretical plan (pre-EUS) and the actual plan (post-EUS). As this was a retrospective study, ability of EUS to alter subsequent therapy and workup was based on a theoretical plan in the absence of EUS (i.e., CT-FNA, mediastinoscopy, or thoracotomy with biopsy to establish the diagnosis) and actual plan (after EUS-FNA). The latter included the diagnosis of malignancy with avoidance of surgery. Patients with nondiagnostic FNA had, by definition, failure of the study to alter subsequent therapy and workup.

\section{RESULTS}

All 26 patients underwent successful EUS-FNA tissue sampling (two to seven passes/patient). Histopathology was determined to be adequate by a bedside cytopathologist in all cases.

Final patient diagnosis by disease category included five patients with benign/infectious masses, nine with inflammatory masses, and 12 with malignant mediastinal masses (Fig. 1). EUS-FNA was successful in establishing the diagnosis in 21 of 26 patients $(81 \%)$ for all disease categories, including infectious in three of five patients $(60 \%)$, benign/inflamma-

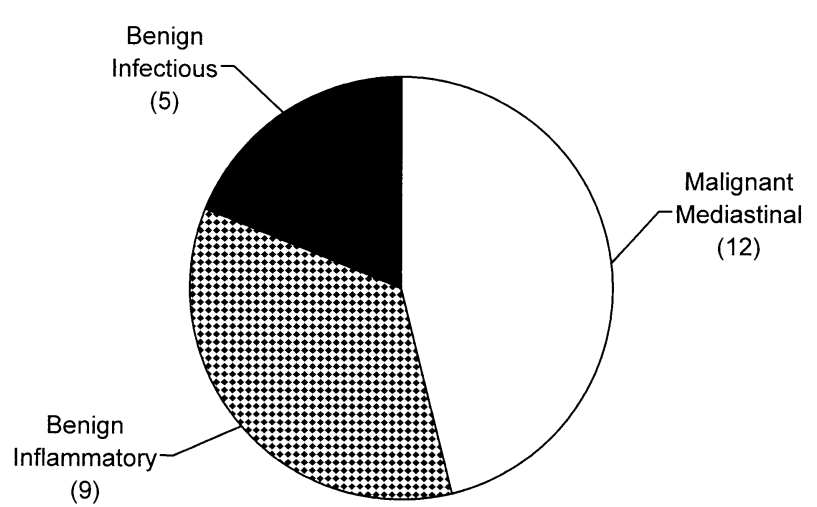

Figure 1. Mediastinal masses of unknown cause: final diagnosis.

tory in seven of nine patients (78\%), and malignant mediastinal in 11 of 12 patients (92\%). Final diagnosis by histopathology and special stains (Table 1) included the following: 1) infectious in five patients, including two abscess, two histoplasmosis, and one tuberculosis (by acid-fast staining); 2) benign/inflammatory in nine patients, including five cysts (bronchogenic and duplication), three fibrosis, and two nonspecific inflammatory masses; 3) malignant mediastinal (12 patients), including two adenocarcinoma of unknown primary (Fig. 2-4), four metastatic lung cancer (non-small cell and small cell), three lymphoma (Fig. 5-7), two metastatic (tracheal and esophageal), and one papillary carcinoma of thyroid. Patients with lymphoma were established by presence of characteristic, small, morphologically identical lymphocytes with high nuclear to cytoplasmic ratio. Because this was suspicious for lymphoma, the bedside cytopathologist requested samples for flow cytometry.

The five patients with duplication and bronchogenic cysts had classic appearance by endosonography (anechoic, thin walled structures adjacent to the esophageal lumen). FNA of the lesion, specifically the wall, and aspiration of fluid was performed for cytologic analysis given the presenting symptoms (including weight loss, chest pain, or fever). The ability of EUS-FNA to alter subsequent workup included three of five patients with infectious mediastinal masses $(60 \%)$, six of nine patients with benign/inflammatory mediastinal masses (67\%), and 11 of 12 patients with malignant mediastinal masses (92\%). The ability of EUS-FNA to alter subsequent therapy included three of five patients with infectious mediastinal masses $(60 \%)$, seven of nine patients with benign/inflammatory mediastinal masses (78\%), and

Table 1. Ability of EUS-FNA to Alter Subsequent Workup and Therapy

\begin{tabular}{lccc}
\hline & Patients & \multicolumn{2}{c}{ EUS Final Diagnosis (\%) } \\
\cline { 3 - 4 } Disease Classification & $(\mathrm{n})$ & Alters Workup & Alters Therapy \\
\hline Infectious & 5 & $3(60 \%)$ & $3(60 \%)$ \\
Benign/inflammatory & 9 & $6(67 \%)$ & $7(78 \%)$ \\
Malignant mediastinal & 12 & $11(92 \%)$ & $9(75 \%)$ \\
Total & 26 & $20(77 \%)$ & $19(73 \%)$ \\
\hline
\end{tabular}




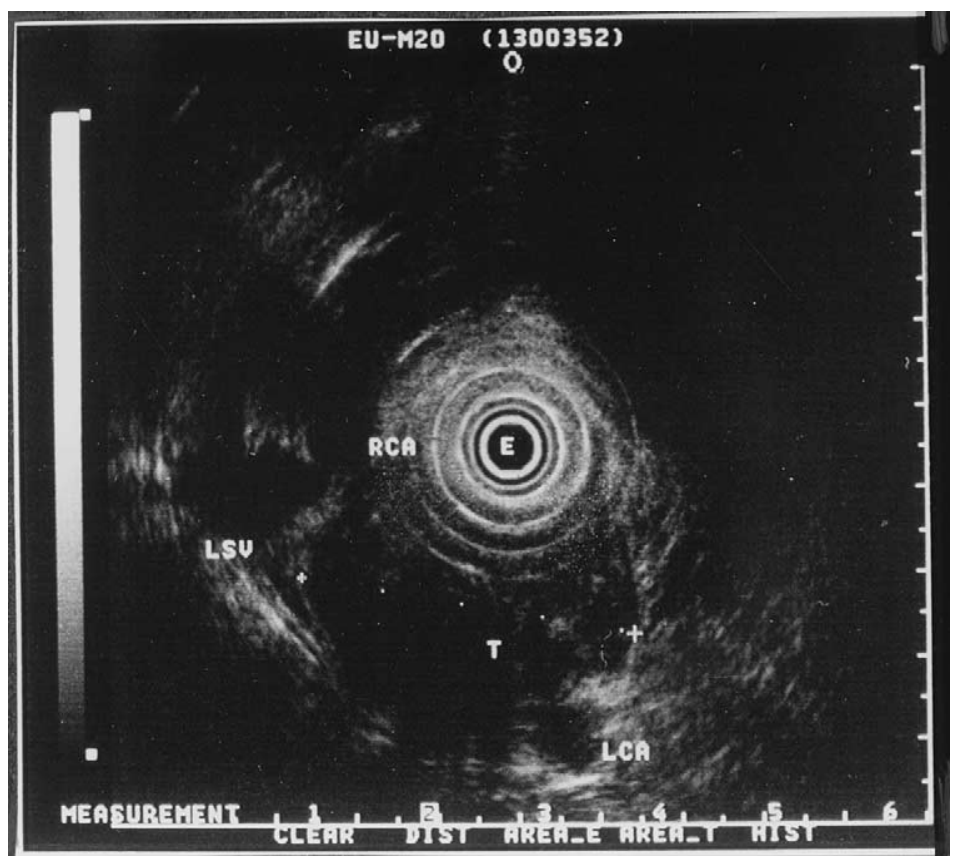

Figure 2. EUS demonstrating 4.2-cm mediastinal mass adjacent to the esophagus at $25-\mathrm{cm}$. LCA $=$ left carotid artery; LSV $=$ left subclavian vein; RCA = right carotid artery.

nine of 12 patients with malignant mediastinal masses (75\%) (Table 1).

EUS-FNA passes (mean) for adequate tissue sampling was lower in nonmalignant disease categories (3.0 and 3.4) versus malignant disease (4.4) but did not reach statistical significance. No complications were seen during the course of the study.

\section{DISCUSSION}

Mediastinal mass lesions represent a heterogenous group of tumors that can involve various components of the mediastinal space. They are most frequently located in the anterosuperior mediastinum (54\%), with the posterior (26\%) and middle mediastinum (20\%) being less frequently involved.

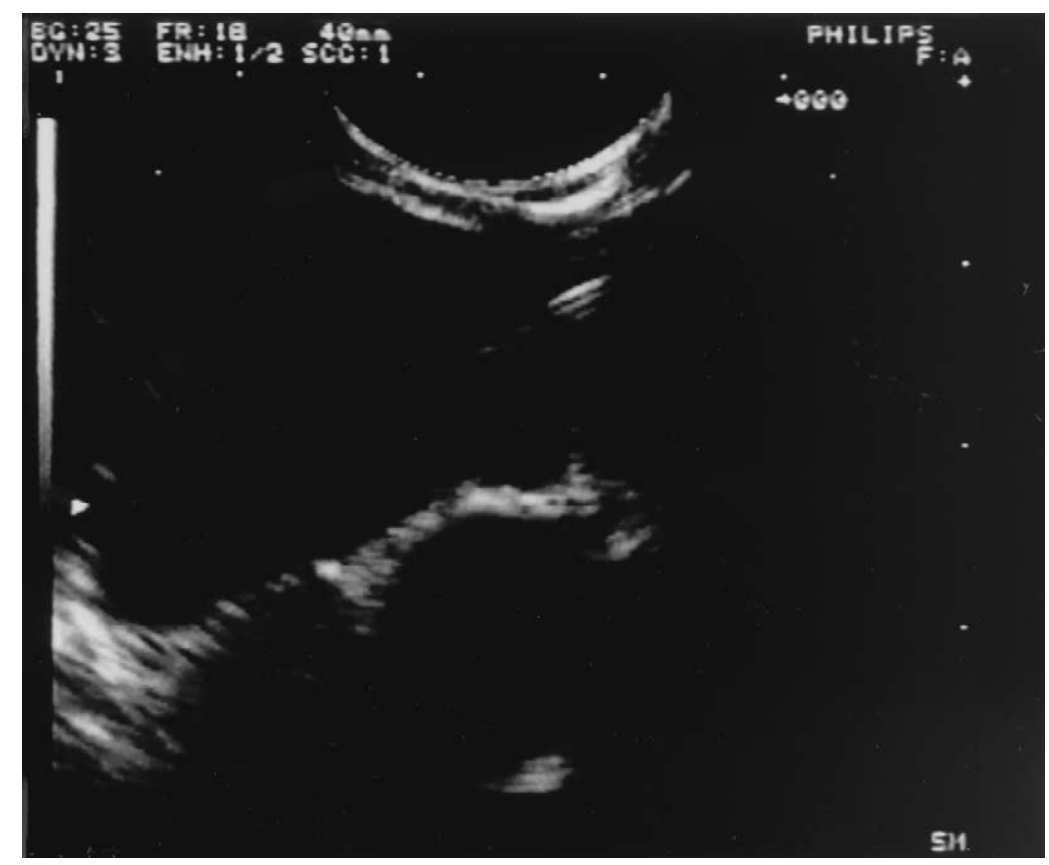

Figure 3. EUS-FNA of patient in Figure 1. 


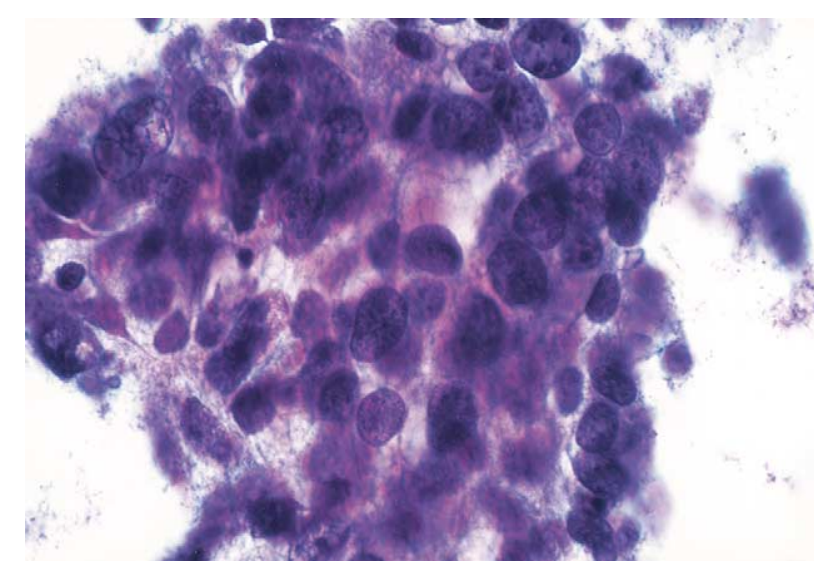

Figure 4. Histology of patient in Figures 1 and 2 demonstrating adenocarcinoma.

The masses that occur most commonly in each of the three anatomic subdivisions and the relative incidence with which they occur are shown in Table 2 (3). In the anterosuperior mediastinum, the most frequent neoplasms are thymoma (31\%), lymphoma (23\%), and germ cell tumor (17\%). Posterior mediastinal lesions are usually neurogenic tumors (52\%), bronchogenic cysts (22\%), and enteric cysts (7\%). Middle mediastinal masses are usually pericardial cysts (35\%), lymphomas (21\%), and bronchogenic cysts (15\%). Overall, malignant neoplasms represent $25-42 \%$ of mediastinal masses (4). Because of the characteristic location of many mediastinal masses, the site of the mass establishes a useful differential diagnosis that aids in planning the diagnostic evaluation.

Imaging-guided percutaneous transthoracic biopsy has become widely accepted. CT, an effective minimally inva- sive technique for the diagnosis of a variety of intrathoracic lesions that are otherwise not readily accessible, has been the preferred guidance modality for difficult parenchymal, hilar, and mediastinal lesions. The major drawback of CT guided biopsy is the lack of real-time guidance, the need to perform multiple section acquisitions, the longer procedure time, the inaccessibility of smaller lesions obstructed by overlying bone, and a higher frequency of pneumothorax.

Alternatives to CT are more invasive. These include thoracoscopy, limited thoracotomy, cervical mediastinoscopy, anterior mediastinoscopy, bronchoscopy with transbronchial needle biopsy, and, more recently, EUS-FNA. Cervical mediastinoscopy has traditionally been considered as the "gold standard" complement to CT in the preoperative staging of the mediastinum in non-small cell lung carcinoma. It provides access to paratracheal lymph nodes and, to a lesser extent, subcarinal lymph nodes. The major limitations are the poorly accessed aortopulmonary region, subcarinal lymph nodes, and the inferior mediastinum. Significant morbidity limits this high risk procedure. General anesthesia is preferred by the thoracic surgeon to provide better control and flexibility in terms of surgical manipulation. Anterior mediastinal masses may cause airway obstruction and cardiovascular collapse after induction of anesthesia. Contraindications include superior vena cava obstruction, tracheal deviation, and aneurysm of the thoracic aorta. Complications occur in up to $3.0 \%$. Hemorrhage and pneumothorax are the most common. Not uncommon are recurrent laryngeal nerve injury, mediastinitis, hemothorax, and chylothorax (5). Inpatient recovery and cost further limit this modality.

Recently, it has been shown that EUS may be used to

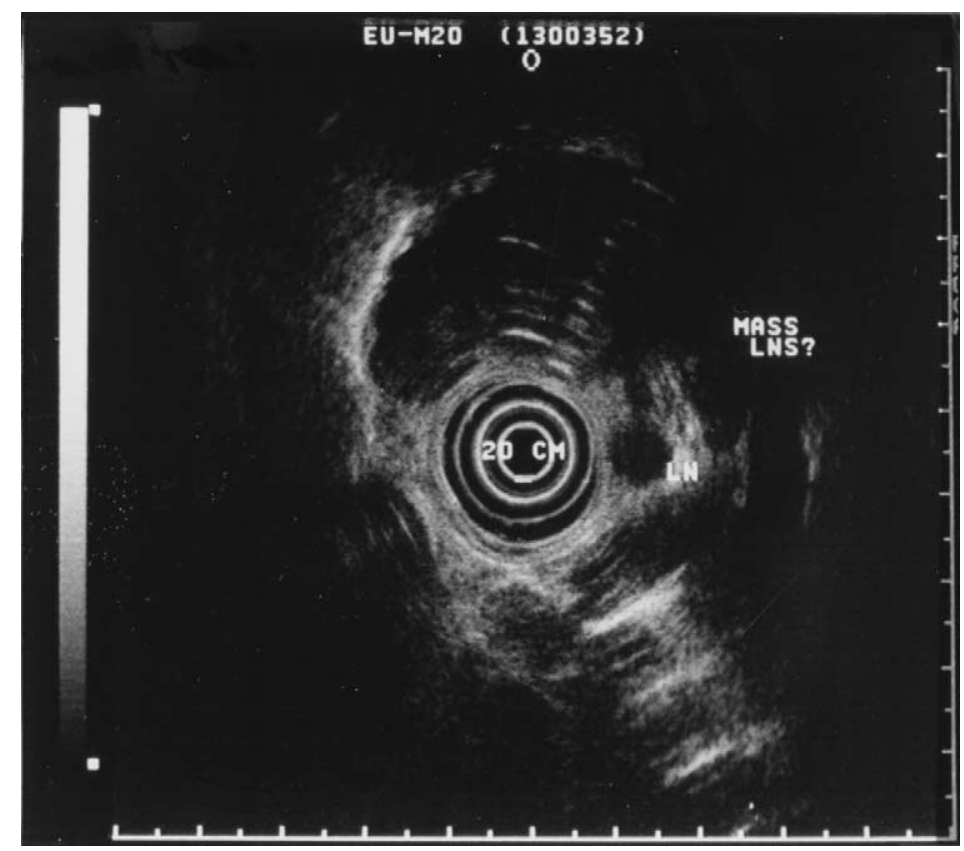

Figure 5. EUS depicting a 4-cm mediastinal mass in a patient presenting with dysphagia and chest pain. 


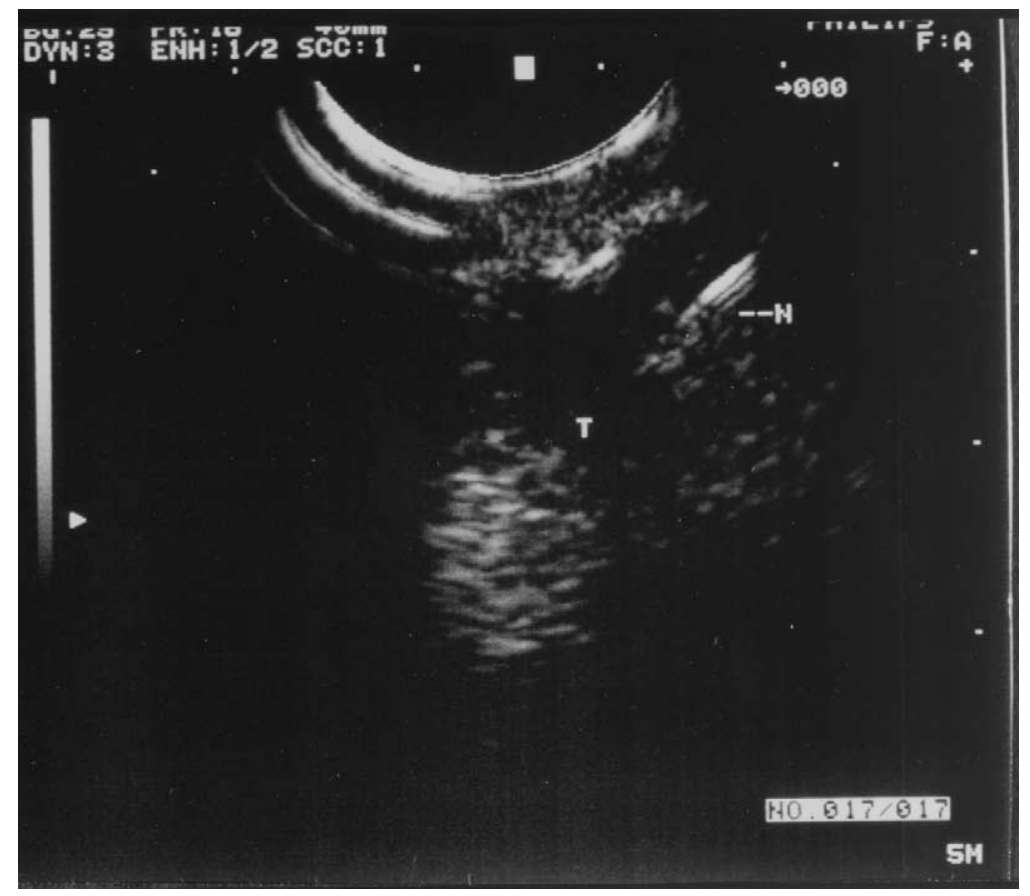

Figure 6. EUS-FNA of patient represented in Figure 4.

image the posterior mediastinum to evaluate periesophageal masses or to detect lymphadenopathy in patients with esophageal or non-small cell lung cancer (6). EUS has been demonstrated to be superior to CT scanning in diagnosing local metastatic lymph nodes in patients with esophageal cancer $(7,8)$. EUS-FNA has also been shown to detect mediastinal sarcoidosis, silicoanthracosis, histoplasmosis, and retrosternal goiters (9).

Unlike previously published articles on EUS-FNA of mediastinal masses and lymph nodes (2, 4, 10-12), our report details the evaluation and tissue sampling of nongastrointestinal lesions. Although some diagnoses demonstrated lymphatic origin, all cases had pre-EUS CT describing the mass lesions in the absence of lymphadenopathy. The above-mentioned articles (2, 4, 10-12) collectively

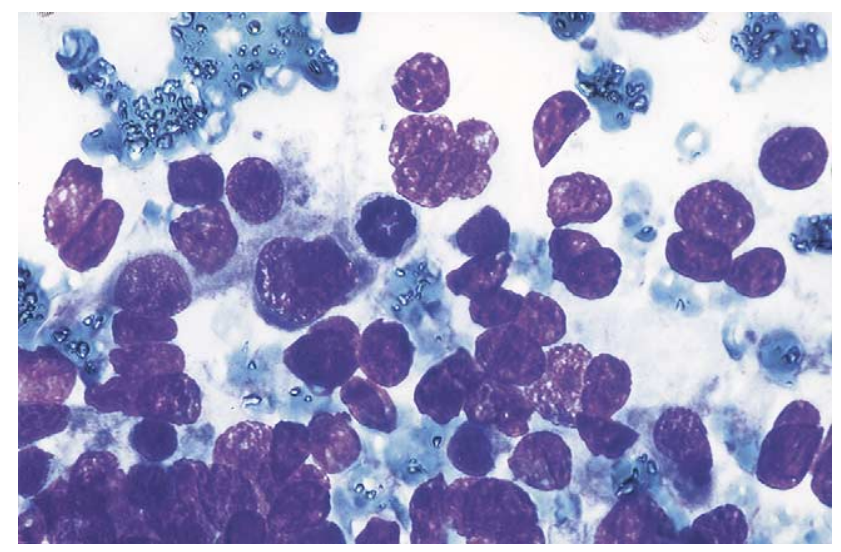

Figure 7. Histology of patient in Figures 4 and 5, demonstrating large cell lymphoma. reported on only 29 patients with mediastinal masses, a number similar to that in our current report.

In a comprehensive report on EUS-FNA, Giovannini et al. (4) described the results of 141 consecutive patients, which included 18 with mediastinal masses. The accuracy for this indication was reportedly $89 \%$. In their report, Hunerbein et al. (2) presented 25 patients with mediastinal lesions, including six patients with nonlymph node masses, all correctly diagnosed by EUS-FNA. Unlike our study, no attempt was made to describe the impact of accurate EUS

Table 2. Anatomic Location of Primary Tumors and Cysts of the Mediastinum

\begin{tabular}{lc}
\hline \multicolumn{1}{c}{ Type of Tumor/Cyst } & Percentage \\
\hline Anterosuperior mediastinum & \\
Thymic neoplasms & 31 \\
Lymphomas & 23 \\
Germ cell tumors (benign and malignant) & 17 \\
Carcinoma & 13 \\
Cysts & 6 \\
Mesenchymal & 4 \\
Endocrine & 5 \\
Other & 1 \\
Middle mediastinum & \\
Cysts & 61 \\
Lymphomas & 20 \\
Mesenchymal & 8 \\
Carcinoma & 6 \\
Other & 5 \\
Posterior mediastinum & \\
Neurogenic & 52 \\
Cysts & 32 \\
Mesenchymal & 10 \\
Other & 6 \\
\hline
\end{tabular}


Table 3. Density and Topographical Approach for Classifying Mediastinal Masses

\begin{tabular}{lllll}
\hline Location & \multicolumn{1}{c}{ Lipidic } & \multicolumn{1}{c}{ Liquid } & Vascular & Soft Tissue \\
\hline AM & Thymolipoma & Thymic/Pleural/pericardic cysts & & $\begin{array}{l}\text { Goiter adenoma } \\
\text { Thymoma }\end{array}$ \\
MM & Neurinoma, neurofibroma & $\begin{array}{l}\text { Bronchogenic cyst } \\
\text { Meningocele }\end{array}$ & $\begin{array}{c}\text { Aortic arch aneurysm } \\
\text { Descending aortic } \\
\text { aneurysm }\end{array}$ & $\begin{array}{c}\text { Lymode } \\
\text { Ganglioma }\end{array}$ \\
Any & Lipomatosis, teratoma & Hydatic cyst & Melanoma & $\begin{array}{c}\text { Lymph node } \\
\text { mediastinitis }\end{array}$ \\
\hline
\end{tabular}

$\mathrm{AM}=$ Anterior mediastinum; Misc $=$ miscellaneous; $\mathrm{MM}=$ middle mediastinum; $\mathrm{PM}=$ posterior mediastinum.

tissue sampling. Several unique features of endosonography make it favorable when specifically compared with CT imaging. Real-time visualization during the procedure permits continuous imaging assessment. EUS uses a multiplanar capability, which allows use of an oblique, angled approach if necessary. CT is limited somewhat by volume effects that occur because of discrete scan thickness. This is exemplified when attempting to visualize the region of the left atrium and the aortopulmonary window. In addition, problems with inadequate contrast or streak artifact generated from soft tissue-air interfaces with the main bronchi and trachea are not present with endosonography. Additionally, the principal complication of CT-guided biopsy is pneumothorax occurring in $25-43 \%$ of patients, which is somewhat higher than the average rate of approximately 25-30\% after fluoroscopically guided thoracic biopsy (13).

In a recent report by Panelli et al. (14), EUS was described to assist in the evaluation of mediastinal masses in 33 patients. Of these, only 25 had EUS-FNA. Three of these had a diagnosis of malignancy established by standard endoscopic biopsy, whereas eight others had FNA of malignant mediastinal lymph nodes. The remaining 14 patients had mediastinal masses diagnosed by EUS-FNA. The authors postulated that EUS-FNA provided a less invasive modality in establishing the diagnosis of mediastinal masses.

We successfully used EUS-FNA to obtain diagnostic biopsies in 26 consecutive patients with idiopathic mediastinal masses. Our results show that EUS-FNA is a safe and effective guidance modality for transesophageal biopsy of mediastinal lesions. In fact, we were able to perform EUSFNA with an overall accuracy of $81 \%$ for defining the cause of the mediastinal mass. A recent study to evaluate nodal spread in non-small cell lung cancer used EUS-FNA to accurately differentiate benign from malignant nodal involvement in $84 \%$ of patients (6). These recent descriptions of real-time evaluation of mediastinal lesions further strengthen the clinical utility of this imaging technique.

Performance of EUS-FNA relies heavily upon skilled endosonographers. A fundamental knowledge of the mediastinal anatomy and pathology that are unique to each compartment may help in determining the etiology (Table 3). With the exclusion of sampling error, it is unclear why the yield for malignant mediastinal lesions was higher than that for the benign/inflammatory or the infectious groups. This may have been the result of more FNA needle passes in malignant (mean 4.4) compared with benign (mean 3 and 3.4) disease categories. It is our experience that in patients with high index of suspicion for malignancy, either on the basis of clinical presentation or atypical cells obtained on initial FNA passes, more aggressive tissue sampling is performed. In our experience, FNA of any organ or tissue should be performed with an attendant cytopathologist or cytotechnologist who can readily examine specimens as they are aspirated. It is generally agreed that immediate feedback regarding the adequacy of aspirated materials for pathological diagnosis decreases the chance of a false negative diagnosis.

We determined that the ability of EUS-FNA affected subsequent workup and therapy in $73 \%$ and $71 \%$ of patients, respectively. No complications occurred as a result of EUSFNA. Specifically, there were no cases of mediastinitis as a result of transesophageal bacterial migration, contrary to what one might expect. Furthermore, no patients received prophylactic antibiotics, including the two patients with mediastinal abscesses. Although we cannot recommend the routine use of antibiotics, it seems prudent to use prophylactic antibiotics if the preprocedural index of suspicion for bacterial contamination is high (i.e., cystic lesions).

Perhaps the only limitation of EUS are the regions of the anterior mediastinum and the contralateral trachea, both of which are of primary concern in patients with suspected lung cancer. This may have an impact on the role of EUSFNA in preoperative staging of the mediastinum. Therefore, EUS-FNA should be considered in all patients with suspicious posterior mediastinal lesions and in those patients with an indeterminate result on CT (13). Anterior mediastinal lesions can be visualized in contrast to lymph nodes in this area because of marked size differences, and these masses are invariably paraesophageal (no air artifact between lesion and esophageal wall). Mediastinoscopy should be reserved for patients with enlarged anterior masses or for patients with suspicious lesions that are not successfully sampled by EUS.

The current role of EUS-FNA to assess the mediastinum is limited by the availability of this application, combined with a lack of awareness of the technique on the part of thoracic surgeons and pulmonologists. As clinical algorithms regarding specific indications validate this approach, 
it is likely that EUS-FNA of idiopathic mediastinal lesions will play an increasing role.

Reprint requests and correspondence: Marc F. Catalano, M.D., 2801 W. KK River Parkway, Suite 1030, Milwaukee, WI 53215. Received Feb. 7, 2001; accepted Apr. 26, 2002.

\section{REFERENCES}

1. Hawes RH, Gress F, Kesler KA, et al. Endoscopic ultrasound versus computed tomography in the evaluation of the mediastinum in inpatients with non-small cell lung CA. Endoscopy 1994;26:784-7.

2. Hunerbein M, Chadimi BM, Haensch W, Schlag PM. Transesophageal biopsy of mediastinal and pulmonary tumors by means of endoscopic ultrasound guidance. J Thorac Cardiovasc Surg 1998;116:554-9.

3. Davis RD, Sabistan DC. Primary tumors and cysts of the mediosturum. In: Sabistan DC, Lyerly HK, eds. Textbook of surgery. WB Saunders, 1997:1906-42.

4. Giovannini M, Seitz JF, Monges G, et al. Fine needle aspiration cytology guided by endoscopic ultrasonography: Results in 141 patients. Endoscopy 1995;27:171-7.

5. Eisenkraft JB, Coe NE, Caplin SA, et al. Cervical mediostinoscopy. In: Barash PG, Collen BF, Stoelting RK, eds. Clinical anesthesia. Lippincott: 1999:904-46.

6. Gress F, Sividas TJ, Sandler A, et al. EUS/FNA biopsy guided by ultrasound and $\mathrm{CT}$ in the preop staging of non-small cell lung CA. A comparative study. Ann Intern Med 1997;127: 604-12.

7. Tio TL, Cohen P, Coene PP, et al. Endoscopic ultrasound and computed tomography in staging of esophageal carcinoma. Gastroenterology 1989;96:1478-86.

8. Botet JF, Lightdale CJ, Gellar A. Preoperative staging of esophageal carcinoma: Comparison of endoscopy ultrasound and dynamic CT. Radiology 1991;181:419-25.

9. Wiersema MJ, Chak A, Lehman GA. Mediastinal histoplasmosis: Evaluation by EUS and EUS/FNA biopsy. Gastrointest Endosc 1994;40:78-81.

10. Williams DB, Sahai AV, Aabakken L, et al. Endoscopic ultrasound guided fine needle aspiration biopsy: A large single centre experience. Gut 1999;44:720-6.

11. Wiersema MJ, Harada N, Daiehagh P, et al. Evaluation of mediastinal lymphadenopathy with transesophageal endosonography guided fine needle aspiration biopsy. Acta Endoscopica 1998;28:7-19.

12. Silvestri GA, Hoffman BJ, Bhutani MS, et al. Endoscopic ultrasound with fine needle aspiration in the diagnosis and staging of lung cancer. Ann Thorac Surg 1996;61:1441-6.

13. Gobien RD, Stanley JH, Vujic I, et al. Thoracic biopsy. CT guidance of thin-needle aspiration. Am J Radiology 1984;142: 827-30.

14. Panelli F, Erickson RA, Prasad VM. Evaluation of mediastinal masses by endoscopic ultrasound and ultrasound-guided fine needle aspiration. Am J Gastroenterology 2001;96:401-8. 